\title{
Una experiencia de formación del profesorado basada en las inteligencias múltiples y la Educación Artística
}

\section{A learning experience with pre-service teachers based on multiple intelligences and visual arts education in the Faculty of Education at the University of Barcelona}

\author{
NAYRA LLONCH MOLINA*1 \\ nayra.llonch@didesp.udl.cat \\ Carolina martín Piñol** \\ carolinamartin@ub.edu \\ JOAN SANTACANA MESTRE** \\ jsantacana@ub.edu \\ * Universidad de Lleida, España. \\ **Universidad de Barcelona, España.
}

\section{Resumen:}

En este artículo se presenta una experiencia didáctica llevada a cabo en una asignatura del área de Educación Visual y Plástica del Grado de Educación Primaria de la Universidad de Barcelona. El objetivo principal de la experiencia, que está sustentada en la teoría de las inteligencias múltiples de Howard Gardner, es desarroIlar la capacidad artística del alumnado a través de la metodología participativa y estimuladora de la creación de material didáctico. El trabajo descrito partió de un estudio previo de las motivaciones del alumnado y de las inteligencias con las que se identificaban. La experiencia ha consolidado una estrategia metodológica basada en las motivaciones del alumnado,

\begin{abstract}
Abstrac:
This article presents a teaching and learning experience carried out in a subject related to the Visual Arts Education area of the Primary Education Degree at the University of Barcelona. The aim of the experience, which is supported by Howard Gardner's theory of multiple intelligences, is to develop students' artistic abilities through the participatory methodology of creating teaching materials. The study described is based on an earlier study of the students' motivations as well as the intelligences with which they identify themselves. The experience has established a methodological strategy for the emerging field of Visual Arts Education, a strategy based on students' motivations, multiple
\end{abstract}

1 Dirección para correspondencia (correspondence address):

Nayra Llonch Molina. Departamento de Didácticas Específicas. Universidad de Lleida.

Av. de l'Estudi General, 4. 25001 Lleida (España). 
Una experiencia de formación del profesorado basada en las inteligencias múltiples y la Educación Artística

Nayra llonch molina, Carolina martín PIÑOl y JOAN SANTACANA MESTRE

las inteligencias múltiples, la interdisciplinariedad y la creatividad en un campo emergente como es el de la didáctica de las Artes Visuales. Además, se presenta un modelo para la didáctica de la Educación Visual y Plástica susceptible de ser aplicado en Educación Primaria, Secundaria y Universitaria.

Palabras clave:

eEducación visual y plástic; inteligencias múltiple; creativida; interdisciplinarieda; modelo didáctico. intelligences, interdisciplinarity and creativity. Furthermore, the study presents a model for the teaching and learning of $\mathrm{Vi}$ sual Arts that can be implemented in Primary Education, as well as Secondary and University Education.

\section{Key words}

Visual arts education; multiple intelligences; creativity; interdisciplinarity; learning model

\section{Résumé:}

Dans cet article nous présentons une expérience didactique réalisée dans la matière d'Éducation Visuelle et Plastique du Grado en Éducation Primaire de l'Université de Barcelone. L'objectif principal de l'expérience qui repose sur la théorie des intelligences multiples de Howard Garder est de développer la capacité artistique de l'élève à travers la méthodologie participative et stimulante de la création de matériel didactique. Le travail décrit part d'une étude préalable sur les motivations des élèves et des intelligences auxquelles ils s'identifient. L'expérience a consolidé une méthodologie basée sur les motivations de l'élève, les intelligences multiples, l'interdisciplinarité et la créativité dans un domaine émergent comme celui de la didactique des Arts Visuels. En outre, nous présentons un modèle pour la didactique de l'Éducation Visuelle et Plastique susceptible d'être appliqué en Education Primaire, Secondaire et Universitaire.

\section{Mots clé :}

Éducation Visuelle et Plastique; intelligences multiples; créativité; interdisciplinarité; modèle didactique.

Fecha de recepción: 3-5-2016

Fecha de aceptación: 2-5-2017

\section{Introducción}

Desde hace décadas el mundo de la educación está mostrando una serie de cambios vinculados con la evolución de las metodologías de la enseñanza, las nuevas tecnologías y de los medios de comunicación (Santacana y Coma, 2014; Santacana y López, 2015). Así también, el mundo de lo artístico, de lo visual y lo plástico ha evolucionado de acuerdo con un nuevo mundo globalizado y ello se pone de manifiesto cuando se revisan sistemas tan sensibles como los de evaluación (Morales, 2001, p. 309-314). Es, por tanto, obvio que si los fundamentos y prácticas de una y otro han sido modificados, parece adecuado que también lo haga el enfoque de la enseñanza y el aprendizaje propios 
de la educación artística en las Facultades de Educación y, por ende, en la escuela primaria, a través de las nuevas generaciones de maestros de primaria: "Una educación artística nueva necesita, por delante de todo lo demás, nuevos profesionales, conocedores de la materia, críticos, conscientes de su hacer político, en consonancia con los tiempos que corren" (Acaso, 2009, p. 123). Por ello, el presente trabajo aborda la enseñanza y el aprendizaje de las artes visuales y plásticas en los futuros educadores de la enseñanza primaria, puesto que son ellas y ellos quienes primero deben familiarizarse con las nuevas tendencias para poder guiar a su alumnado (Báez, 2010). También hay que tener en cuenta las apuestas de la educación artística desde la visión patrimonial (Fontal, Marín y Garcia, 2015) ya que el conocimiento, la comunicación, la comprensión, la creación y la identización (Gómez Redondo, 2013) son dimensiones propias de la educación artística pero también del patrimonio cultural. Por otro lado, debemos considerar que éste forma parte de la identidad de nuestros alumnos, por lo que se configura no solo como contenido clave de la educación artística -como patrimonio artístico y cultural- sino también como un modo de enfocar el material (Fontal, 2008). En relación con esta tendencia, el presente trabajo muestra una propuesta didáctica cuya potencialidad es que enlaza con otras iniciativas que persiguen una enseñanza de la Educación Artística que potencie el aprendizaje de los estudiantes a través de un planteamiento que bebe de metodologías procedentes de la didáctica de las ciencias sociales y de la educación patrimonial, una vez planteada conjuntamente con el proceso de creación que pone en juego materiales diferentes y que estimula aquellas capacidades (inteligencias) en las que cada uno/a de los alumnos/as se siente más cómodo.

En realidad, la propuesta que presentamos trata de ofrecer la oportunidad de iniciarse en la educación artística desde una vertiente vinculada con la educación del patrimonio, que no deja de ser parte imprescindible de educación de las personas y al que se hace referencia en multitud de ocasiones en el currícula (como en "conocer, comprender y respetar el patrimonio"). Se trata de huir de, en palabras de Acaso (2013, p. 31) de "la pedagogía tóxica que envenena el conocimiento propio, y que nos ha hecho creer que es viable un anclaje completo, una reproducción fiel y total entre lo que enseña el profesor y lo que aprende el estudiante". 


\section{Marco teórico}

\subsection{Algunos parámetros de la Didáctica de la Educación Visual y Plástica}

Lo primero que cabe decir es que la experiencia y el estudio de la expresión artística muestra que la mayoría de actividades que se desarrollan, tanto en las aulas de las escuelas de primaria como en las propias facultades de Educación del Estado, poseen un cierto carácter fragmentario y a menudo se conciben como actividades aisladas sin relación entre sí; parece como si los objetivos de cada actividad finalizaran en ella misma. Tal y como plantea Acaso (2013), hay una confusión muy extendida entre la EVP y el concepto de manualidades. En realidad, las manualidades, como su propio nombre indica, se nos aparecen como un auténtico pasatiempo en el que se realizan cosas con las manos, por lo que los únicos procesos que se desarrollan en la ejecución de la obras son aquellos enfocados en la producción, dejando de lado el tan importante proceso y el análisis. Dicho resultado parece que deba cumplir premisas fundamentales que se convierten en tópicos: ha de ser bello, bonito, ornamental, bien acabado, que decore... Es obvio que para producir esta cantidad de objetos y con estas características, los contenidos se centran en la enseñanza y en el aprendizaje de diferentes técnicas y requiere de una asimilación y una producción en la mayoría de ocasiones rápida y con un objetivo.

Este planteamiento también viene agudizado por lo que Efland (2002) expone como el drama de la educación artística, la dualidad entre los que defienden la enseñanza de la educación artística como una disciplina más, siguiendo los esquemas propios centrados en los contenidos, y los que se decantan por el abandono de todo método en pro de la expresión personal, dejando varada la enseñanza artística en un descubrimiento a través de los propios medios de cada niño.

Este panorama dentro del área de expresión plástica probablemente es el resultado de la falta de conocimiento por parte de muchos educadores de planteamiento metodológicos y modelos de aprendizaje. En realidad, dichos planteamientos resultan complejos por el hecho que dentro de un área tan amplia caben infinidad de métodos. Quizás esta dispersión metodológica es lo que diferencia esta área de aquellas cuyos conocimientos necesariamente requieren de una estructura acumulativa y ordenada. 
Contra esta fragmentación metodológica poco puede hacerse desde la didáctica, dado que todo planteamiento didáctico requiere de un método, por lo que en la presente propuesta se apuesta por un planteamiento educativo basado en el paradigma humanista, es decir, desde la comprensión del alumno como centro de los procesos de enseñanza-aprendizaje, por tanto, desde una visión integral (Fontal, Marín y García, 2015). Afortunadamente, la educación artística se está modificando como consecuencia del cambio de enfoque de la didáctica las artes visuales y de las reformas educativas generales, un viraje que comenzó hace más de tres decenios, y que tal y como Larenas (2005) recuerda supone concebir el currículo como un proceso creativo y un espacio conceptual en el cual los estudiantes desarrollan sus ideas con la ayuda de los educadores, quienes no actúan meramente como guías, sino como compañeros críticos. Desde esta perspectiva, el currículum es entendido como una forma de mediación entre estudiantes y profesores (p. 79).

La idea, es pues, que los discentes agudicen su delicadeza respecto a los materiales y a las posibilidades de éstos, tal y como Eisner (2004) propone: "el hecho de aprender a abordar tareas como éstas, que tienen un número infinito de posibles soluciones, prepara a los estudiantes para otras tareas más importantes desde el punto de vista social en una variedad de campos" (p. 52).

Con el presente planteamiento, se busca también atacar a una de las falsas creencias tan extendidas y también planteadas por Fontal, Marín y García (2015) en que la atribución del éxito artístico parece un don el que se nace y que condena a la mayoría de la población -frente a una minoría que se cree que es poseedora del genio artístico. En esta propuesta la importancia reside en poder crear material didáctico patrimonial a través de la IM en la que uno se encuentra más cómodo, aquí no cabe la sensación de no haber sido tocado por los dioses.

\subsection{La vigencia de la teoría de las inteligencias múltiples}

Cuando se plantea la didáctica de una disciplina, normalmente, se tiene en cuenta que nuestros sistemas de conocimiento se basan en sistemas simbólicos que hay que ir aprendiendo (Piaget, 1961). Así, los planteamientos de autores como Gardner (1994), básico para el presente trabajo, basan la identificación de la inteligencia -o de las inteligencias- en que 
Una experiencia de formación del profesorado basada en las inteligencias múltiples y la Educación Artística

NAYRa llonch MOlina, Carolina martín PIÑOL y JOAN SANTACANA MESTRE

disciplinas como la lengua, el arte, la música e incluso las matemáticas se fundamentan en sistemas simbólicos propios:

$\mathrm{Si}$, en efecto, se ha especificado la naturaleza de las inteligencias humanas -las materias primas para la cognición- por una parte, y la gama de papeles y funciones culturales humanos por la otra, debiera poder hacerse una lista de todos los sistemas simbólicos posibles y, por así decirlo, de todos los dominios en los cuales los seres humanos se pueden involucrar de manera intelectual. (p. 231)

Hoy en día, más de treinta años después de los primeros trabajos de Gardner en este ámbito, buena parte de las teorías de aprendizaje actuales en disciplinas tales como la educación artística se basan, todavía, en la adquisición de los distintos sistemas simbólicos y en el desarrollo de las inteligencias múltiples (a partir de ahora IM), que han pasado de siete a ocho y posteriormente a nueve (Gardner, 2003).

Sin embargo, el pensamiento psicopedagógico actual está lejos de ser unívoco, autores como Gordon Allport (1959), quien, en su teoría de los rasgos, concibió la mente como varios sistemas que operan de forma paralela e independientemente unos de los otros, y en ningún caso se conciben con un funcionamiento en serie.

Otros autores, como Fodor (1983), apasionado seguidor de Chomsky, explican lo mismo; en el caso de Fodor, a través de la teoría de la modularidad de la mente. En ella, concebía los procesos mentales como auténticos módulos independientes, cada uno de los cuales fusionaba según sus propias reglas. Admitía, además, la existencia de una mente centralizada que, en este caso, no la concebía como encapsulada y le permitía comparar diversas entradas. En este sentido, Gardner $(1994,2003)$ no acepta fácilmente ese cerebro central o esta capacidad supramodular que ordena o combina los módulos de la mente, aunque sí acepta la existencia de módulos más grandes y otros más pequeños. Por ello, cada módulo -él le Ilama "inteligencia"- es algo que puede desarrollarse siempre que reciba los estímulos pertinentes. En el fondo, en la teoría de la IM, Gardner plantea un conjunto de potencialidades intelectuales humanas que todos los individuos pueden tener, pero que a causa de la herencia, la educación y la práctica algunos desarrollan unas más que otras.

Por otro lado, uno de los planteamientos que enfatizan la crítica a la teoría de las IM provienen de Larivée (2010). Este autor plantea su 
posición crítica como una disyuntiva, aun cuando a lo largo del texto realmente no la discute y no llega a ninguna conclusión fundamentada. Quien sí plantea una revisión crítica más contundente es Ibáñez (2010). El primer punto de su revisión crítica se basa en que

las inteligencias de la IM resultan ser proclividades innatas sobre las que actúa la heterogeneidad de los procesos de desarrollo de los individuos, así como las prácticas y valores culturales particulares. Entonces, por la arbitrariedad de sus criterios, la falta de sistematización de sus elementos significativos y su carácter eminentemente tautológico, la IM corresponde mejor a un esquema de identificación y no a una teoría científica. La IM no contiene postulados propios, ni de otras teorías, de los que se pueda inferir cómo se desarrolla -o debe desarrollarse- una determinada competencia intelectual -ya que de por sí se concibe como innata. Tampoco permite predecir si un individuo o clase de individuos sea competente en algún dominio particular antes de nacer o antes de exhibir algún indicio de sus proclividades intelectuales; como esto sólo puede predicarse una vez que ha acontecido el hecho, se demuestra lógicamente que la 'teoría' es irrefutable (p. 139).

El segundo elemento del análisis crítico de lbáñez se basa en el carácter "político" de la inteligencia múltiple, ya que apunta que

por una parte, su propio autor [Gardner] confiesa que el reto más importante que pretendía enfrentar es que tuviera utilidad a los políticos y practicantes que tienen como encargo el desarrollo de los individuos; ofrece un marco de trabajo basado en las inteligencias múltiples que pudiera aplicarse en cualquier situación educativa. El segundo aspecto retoma el carácter irrefutable de la propuesta, al ser lógicamente imposible contradecir con razones de hecho sus aseveraciones (Ibáñez, 2010:139).

Por lo tanto, el análisis crítico de Ibáñez hace hincapié en que la llamada teoría de las IM en realidad no es una teoría -lo cual argumenta sobradamente- y hay que situarla mejor en el terreno metodológico; en realidad, la rebaja al nivel de técnica para la identificación de algunos dominios de cierta importancia social o cultural en los

que puede organizarse el desempeño de los individuos humanos a diferentes niveles, cumpliendo determinados criterios de logro, morfológicos y 
Una experiencia de formación del profesorado basada en las inteligencias múltiples y la Educación Artística

Nayra llonch molina, Carolina martín piñol y JoAn SantaCana mestre

circunstanciales que le dan carácter de 'competencia'. Cuáles son las condiciones que determinan la organización del comportamiento a determinados niveles de competencia en un domino particular es quizás la pregunta central en el tema de la inteligencia, o mejor dicho, del comportamiento inteligente, una pregunta ante la cual la IM enmudece o, en el peor de los casos, da respuestas que cualquier persona enterada de la tradición popular sobre la inteligencia podría contestar (Ibáñez, 2010:139).

A pesar de la validez difícilmente cuestionable de las críticas vertidas, y que lo que Gardner identifica como inteligencias sus críticos lo rebajan a "habilidades" o a "competencias", la crítica que Gardner realiza del modelo vigente de educación se mantiene sólida, ya que en sus trabajos intenta demostrar que es posible una escuela que garantice un aprendizaje y evaluaciones afines al perfil cognitivo de cada alumn/a; y es obvio que para ello hay que cambiar muchas cosas en nuestra escuela. Los planteamiento gardnenianos no solo proponen cambios en la forma de aprender, sino, sobre todo, en cómo evaluar. Así, observa que

Gran parte de nuestros esfuerzos recientes se han encaminado al desarrollo de medios de evaluación que "hagan justicia a la inteligencia": que permitan medir los valores intelectuales sin pasar por la "óptica" del lenguaje y la lógica, como es menester en las mediciones escritas. Al comienzo, juzgamos que sería posible, y deseable, tratar de medir la inteligencia de un individuo en "forma pura", hasta crear algo semejante a un perfil de inteligencia dividido en siete vertientes. Sin embargo, a medida que fuimos aceptando las perspectivas de contextualización y distribución, nos pareció poco recomendable, y acaso imposible, intentar medir la inteligencia "pura" (Gardner, 1994:8).

\section{Y continúa diciendo que}

De hecho, pienso que no es posible medir las inteligencias en forma pura, y los tipos de evaluación que apoyo son del todo distintos de los que se relacionan con las pruebas del coeficiente de inteligencia. Me opongo a los esfuerzos por caracterizar a los individuos o a los grupos como si presentaran uno u otro perfil de inteligencia. Si bien en cualquier momento una persona o un grupo puede manifestar determinadas inteligencias, se trata de una imagen cambiante (p. 12). 
Como hemos visto, su planteamiento es que cada alumno/a debe ser evaluado según su propio perfil, una propuesta muy sugestiva siempre que se mueva en el marco estricto del sentido común, dado que un modelo de este tipo no es de fácil aplicación sin recursos económicos muy importantes -tan importantes que lo acercan a la utopía. Lo que resulta innegable es que la educación -la escuela- tiene la obligación de proporcionar oportunidades de éxito a todas y todos sus miembros; ciertamente, es posible que personas diversas, por caminos también distintos -llámeseles "inteligencias"-, puedan llegar a comprender un mismo tema. Y ello es así porque cuando cada cual elige su camino la potencia de la motivación juega a su favor, ya que "son aspectos [la motivación y la atención] en extremo importantes de la existencia humana, y que los intentos de adiestrar cualquiera -en realidad, todas- de las inteligencias pueden fracasar si falta la motivación apropiada y la atención suficientemente centrada" (Gardner, 1994:221-222), y si la escuela ha de ser un aprendizaje para la vida, la teoría de las IM todavía tiene mucho que aportar.

La traducción de los planteamientos de Gardner a la escuela, por las razones antedichas, no resulta fácil; sin embargo, si reducimos estos planteamientos a un modelo de intervención aplicado a la educación artística, los resultados pueden ser ampliamente satisfactorios y compartidos por muchos docentes (Luca, 2000; Suazo, 2010; Suárez, Maiz, Meza, 2010).

Finalmente, esta derivación hace que el presente proyecto se comporte como una propuesta educativa Basada en las Artes (Art-Based Educational Research ABER, 2008) pues utiliza elementos artísticos y estéticos. Mientras que la mayoría de la investigación en Humanidades, Ciencias Sociales y Educación utiliza elementos no lingüísticos, relacionados con las artes visuales o performativas (...).

Busca otras maneras de mirar y representar la experiencia. A diferencia de otras perspectivas de investigación la lba no persigue la certeza sino el realce de perspectivas, la señalización de matices y lugares no explorados. Por eso no persigue ofrecer explicaciones sólidas ni realizar predicciones "confiables" sino que pretende otras maneras de ver los fenómenos a los que se dirija el interés del estudio (Hernández, 2008). 


\section{Marco empírico}

\subsection{Objetivos y diseño de la propuesta}

La implementación de elementos propios de la teoría de Gardner en la didáctica Visual y Plástica resultan adaptables siempre que se parta de dos paradigmas complementarios: la teoría del modelo y la teoría de proyectos. Así la presencia de proyectos de filiación artística con un modelo otorga a los artefactos pedagógicos una multiplicidad de planos que los convierten en algo mucho más interesantes, entendiendo como modelo, en su sentido más amplio, una esquematización de la realidad con finalidades aplicativas. Por ello, el modelo no suele ser verdadero o falso, sino simplemente útil o sugerente. La teoría de proyectos, por otra parte, se basa en la suposición que el aprendizaje siempre se realiza mediante círculos concéntricos a partir de un punto central o de interés. Decroly (Muset, 2001) desarrolló de forma magistral la teoría de los centros de interés, que desde la óptica de la modelización tiene grandes similitudes con la teoría de proyectos.

Es por esta razón que los objetivos que se quieren alcanzar con el presente trabajo son, por un lado, y como objetivo general, desarrollar la capacidad artística, creativa y de interpretación a lo largo del proceso de enseñanza aprendizaje a través de la metodología participativa y estimuladora de la creación de material didáctico sustentada en la propuesta de las IM de Gardner

Por otro lado, se plantean los siguientes objetivos específicos

1. Detectar las motivaciones del alumnado que cursa la asignatura de Didáctica de la EVP en la Facultad de Educación como asignatura obligatoria del Grado en Primaria.

2. Consolidar una estrategia metodológica a través de una intervención didáctica para el desarrollo de la capacidad artística entre las IM y la enseñanza de las Artes Visuales.

\subsection{Muestra}

La experiencia que a continuación describimos se enmarca precisamente en este paradigma didáctico; el marco en el cual se desarrolla la experiencia es la Facultad de Educación de la Universidad de Barcelona (UB) y pivota alrededor de la experiencia de tres profesores pertenecientes 
al Departamento Didàctica de las Ciencias Socieles, de la Educación Musical, de la Educación Física y de la Educación Visual y Plástica (UB y UDL) pertenecientes al mismo grupo de investigación especializado en didáctica del patrimonio.

El análisis del contexto se basa en el análisis y la intervención sobre tres grupos de la asignatura de Educación Visual y Plástica del Grado de Educación Primaria de la Facultad de Educación de la Universidad de Barcelona (al que nos referiremos a partir de ahora como GEP) a lo largo de tres cursos académicos consecutivos (2012-2013, 2013-2014 y 20142015). La asignatura, de 6 créditos y, por tanto, de 60 horas de docencia, se enmarca en el primer semestre del segundo curso del GEP de la UB. Cabe señalar, por tanto, que estamos hablando de una muestra con un nivel muy semejante entre ellos/as, alumnos/as con el deseo de formarse como maestros de primaria, aún con poca formación, pero con el criterio necesario para desear crecer en cuanto a metodologías vinculadas a la didáctica en general y que, en una multitud de casos, su cultura artística y plástica, así como la diversificación de recursos vinculados a ella, se remonta a su propia experiencia en primaria, secundaria o bachillerato.

El primer grupo de la asignatura EVP en que se planteó la propuesta, constaba de 30 alumnos/as matriculados (curso 2012-2013), el segundo grupo era de 32 alumnos/as (curso 2013-2014), y, finalmente, en el curso 2014-2015 se repitió la experiencia con un grupo más poblado que constaba de 42 alumnos/as. Referente a las edades del alumnado sujeto al análisis estamos hablando de estudiantes cuyo rango de edad varía entre 19 y 22 años, principalmente, y con un porcentaje mucho más alto de población femenina (87.1\%).

Para dar inicio a la propuesta, y con el fin de poder motivar lo máximo posible su desarrollo, se realizó un primer análisis con el objetivo de detectar motivaciones, deseos e incluso frustraciones respecto a la asignatura vinculada con la Didáctica de la EVP en Primaria, así como detectar también si el alumnado era consciente de la teoría de las IM y/o de aquellas inteligencias que tenía más desarrolladas.

\subsection{Encuestas previas y sus resultados}

El diseño de la encuesta se determinó a través de categorías y estas se desarrollaron a través de cuatro cuestiones que se presentaban al alumnado en la primera sesión de clase de cada uno de los tres cursos aca- 
démicos. Para la recolección de información se empleó una entrevista semi-estructurada y con cuatro preguntas abiertas. Dado el carácter cualitativo de la investigación, cabía la posibilidad de crear más categorías y ahondar en aquellos aspectos como las formas de pensamiento, actitudes, experiencias y hechos concretos relacionados con la enseñanza de las Artes Visuales y Plásticas y/o de las IM.

En la tabla 1 mostramos aquellas categorías que se plantearon, así como los objetivos de las mismas y la formulación de las cuestiones que se entregaron al alumnado.

Tabla 1. Categorías, objetivos y formulación de preguntas de las cuestiones de la encuesta previa.

\begin{tabular}{|c|c|c|c|}
\hline & Categoría & Objetivo a alcanzar & $\begin{array}{l}\text { Formulación de la cues- } \\
\text { tión }\end{array}$ \\
\hline $\begin{array}{l}\text { Cuestión } \\
1\end{array}$ & Motivaciones & $\begin{array}{l}\text { Detectar todas aque- } \\
\text { Ilas motivaciones que } \\
\text { el alumnado tiene } \\
\text { acerca de la asignatu- } \\
\text { ra. Localizar aquellos } \\
\text { miedos, frustraciones / } \\
\text { o logros del alumnado } \\
\text { en relación a la cultu- } \\
\text { ra visual y plástica. }\end{array}$ & $\begin{array}{l}\text { ¿Qué experiencia (positiva } \\
\text { o negativa) tienes en rela- } \\
\text { ción con todo lo referido al } \\
\text { concepto global de Visual } \\
\text { y Plástica? }\end{array}$ \\
\hline $\begin{array}{l}\text { Cuestión } \\
2\end{array}$ & $\begin{array}{l}\text { Conocimien- } \\
\text { to acerca de } \\
\text { planteamien- } \\
\text { tos teóricos y } \\
\text { didácticos de } \\
\text { estrategias me- } \\
\text { todológicas }\end{array}$ & $\begin{array}{l}\text { Detectar los plantea- } \\
\text { mientos teóricos y/o } \\
\text { estrategias metodoló- } \\
\text { gicas de los que están } \\
\text { dotados. }\end{array}$ & $\begin{array}{l}\text { ¿Qué planteamientos crees } \\
\text { que son los óptimos para } \\
\text { desarrollar las clases de vi- } \\
\text { sual y plástica (estrategias, } \\
\text { material, condiciones del } \\
\text { espacio...)? Describe cuá- } \\
\text { les son. }\end{array}$ \\
\hline $\begin{array}{l}\text { Cuestión } \\
3\end{array}$ & $\begin{array}{l}\text { Técnicas artís- } \\
\text { ticas }\end{array}$ & $\begin{array}{l}\text { Detectar qué dominio } \\
\text { tienen de las técnicas } \\
\text { artísticas. }\end{array}$ & $\begin{array}{l}\text { ¿Existe alguna técnica artís- } \\
\text { tica que despierte un inte- } \\
\text { rés especial en ti? }\end{array}$ \\
\hline $\begin{array}{l}\text { Cuestión } \\
4\end{array}$ & $\begin{array}{l}\text { Inteligencias } \\
\text { múltiples }\end{array}$ & $\begin{array}{l}\text { Analizar si el alumna- } \\
\text { do es consciente de } \\
\text { que existen distintas } \\
\text { inteligencias y si es } \\
\text { así, detectar cuál es la } \\
\text { que poseen en mayor } \\
\text { medida. }\end{array}$ & $\begin{array}{l}\text { De todas estas capacida- } \\
\text { des, habilidades (inteligen- } \\
\text { cias: musical, lingüística, } \\
\text { lógico matemática, espa- } \\
\text { cial, corporal cenestésica, } \\
\text { interpersonal, intraperso- } \\
\text { nal, naturalista). ¿Cuál/es } \\
\text { crees que has desarrollado } \\
\text { a lo largo de tu vida con } \\
\text { más intensidad? }\end{array}$ \\
\hline
\end{tabular}


En realidad, el análisis de los resultados de los tres grupos, con una muestra de 104 estudiantes, se realizaba previamente al planteamiento de la propuesta anual para detectar si el grupo clase tenía unas características muy distintas al de los otros cursos. Aquí se presentan los resultados transversalmente, buscando la importancia vinculada al planteamiento de la propuesta, ya que esta pretende cubrir la diversidad de los planteamientos que el alumnado revela:

- Respecto a la primera categoría, que trata de detectar todas aqueIlas motivaciones que el alumnado tiene acerca de la asignatura, así como de localizar aquellos miedos, frustraciones o logros del alumnado en relación a la cultura visual y plástica, podemos destacar que las inquietudes de los alumnos/as se centran en aprender cómo enseñar en sus clases. La mayoría de ellos manifiesta la voluntad de un aprendizaje para un futuro desarrollo en sus aulas y aflora el miedo al desconocimiento de una buena metodología para poderlo llevarlo a cabo (cómo enseñar, con qué herramientas, qué materiales emplear y por qué).

- Por otro lado, el alumnado manifiesta el interés por el desarrollo y elaboración de proyectos, así como un gran interés en saber cómo plantear y crear materiales didácticos con una especial voluntad de transversalidad entre materias. En realidad, uno de los objetivos que buscan es el de adquirir unas mayores habilidades para poder crear materiales para otras asignaturas no vinculadas con los términos artísticos pero que sí que sean plásticos y creativos. Así vemos como el alumno 14 manifiesta que: "aprender a saber crear materiales didácticos artísticos que puedan ser utilizados en diversas asignaturas", o como el alumno 38, que tiene como objetivo conseguir y adquirir mediante el "aprendizaje de nuevas didácticas, nuevas técnicas para enseñar, sea la materia que sea, de una manera más creativa, original, transformadora y crítica".

- Todo ello va muy unido a las motivaciones que los estudiantes persiguen, así como a construir su propio discurso con el que poder motivar en un futuro dentro así nos hablan de "aprender maneras divertidas e innovadoras de enseñar" o bien "aprender cómo puedo enseñar contenidos de manera motivadora". Más de una treintena de los encuestados manifiestan que desean aprender a disfrutar del aprendizaje y a motivar y valorar los trabajos de sus futuros alumnos/as. 
- En cuanto al concepto de motivación, también se intercala con las malas experiencias y los miedos que proclaman los estudiantes acerca de su destreza con los materiales plásticos y con prácticas anteriores. Destacamos el comentario del alumno 2, que sorprende por la mala experiencia anterior: "Quiero que la asignatura me motive para que no sea tan aburrida como lo era en la ESO, ya que tengo muy mal recuerdo de esta asignatura".

- Por lo que atañe a la segunda cuestión, referida a los planteamientos acerca del desarrollo de las sesiones de visual y plástica las respuestas son de tipología muy distinta. Entre ellos destaca su preocupación por fomentar la capacidad del alumnado en la creación de obra digital. Más de la mitad, 67 de los 104 alumnos/ as, muestran un especial interés por la alfabetización digital en relación a las técnicas creativas, el diseño gráfico, etc. La mayoría se refiere a dicha inquietud con conceptos diversos como los siguientes "la parte digital artística" (alumno 14); "saber utilizar las TIC y el arte" (alumno 53);"conocer programas informáticos para modificar las imágenes" (alumno 37); "métodos virtuales para crear imágenes en 3D" (alumno 79); "perfeccionar aspectos tecnológicos para crear proyectos creativos" (alumno 98)...

- Respecto a la cuestión referida a los conceptos vinculados con las técnicas artísticas, las respuestas son diversas. De los 104 resultados, 78 muestran un desconocimiento de los contenidos y técnicas con respuestas como "no me acuerdo", "no conozco", "no sé pero estoy dispuesta a probar cualquiera", "no me despierta interés ninguna" (alumnos 23, 58, 73). También desconocen espacios artísticos y culturales, expresando su deseo de "culturizarse y familiarizarse con el mundo artístico" (alumno 68); "mejorar mi capacidad de creación y manipulación (alumno 6) o "adquirir conceptos y competencias en técnicas artísticas". De entre estas respuestas Ilama la atención un concepto que hemos abordado en el marco teórico, el de las "manualidades", ya que 20 de los 104 se refieren a las técnicas artísticas como "manualidades" y/o "saber dibujar". También cabe destacar que una minoría se refiere a ciertas técnicas pero que dejan entrever el desconocimiento de las mismas por cómo se refieren a ellas con conceptos amplios o inexactos como "pintura", "dibujo con boli", "cómic" (alumnos $64,58,97)$. 
- En relación a las IM, casi la totalidad del alumnado (100 de 104) conocía la teoría de las IM de Gardner; solo 4 de los alumnos/as respondieron con conceptos no vinculados a ella. Éstos (alumnos $7,28,79$ y 101) respondieron a la cuestión con conceptos vinculados a la "capacidad creativa" o "capacidad imaginativa" sin ser capaces de definir cuál de las inteligencias tenía más desarrollada.

- En referencia al resto del alumnado, los resultados (expuestos en el Fig. 1) muestran cómo el tanto por ciento más elevado escogieron la inteligencia interpersonal (32\%) sin embargo la inteligencia intrapersonal, característica de aquellas personas que se dedican a la docencia, solo obtuvo un $12 \%$ de los resultados. Tras la que obtuvo más puntuación, se detectan la inteligencia corporal-cenestésica y la musical, ambas con un $16 \%$. Para finalizar, la inteligencia lógico matemática (10\%) así como la naturalista y la lingüística (con un $7 \%$ ) son aquellas menos representativas dentro de los grupos clase. La espacial no obtuvo ningún resultado.

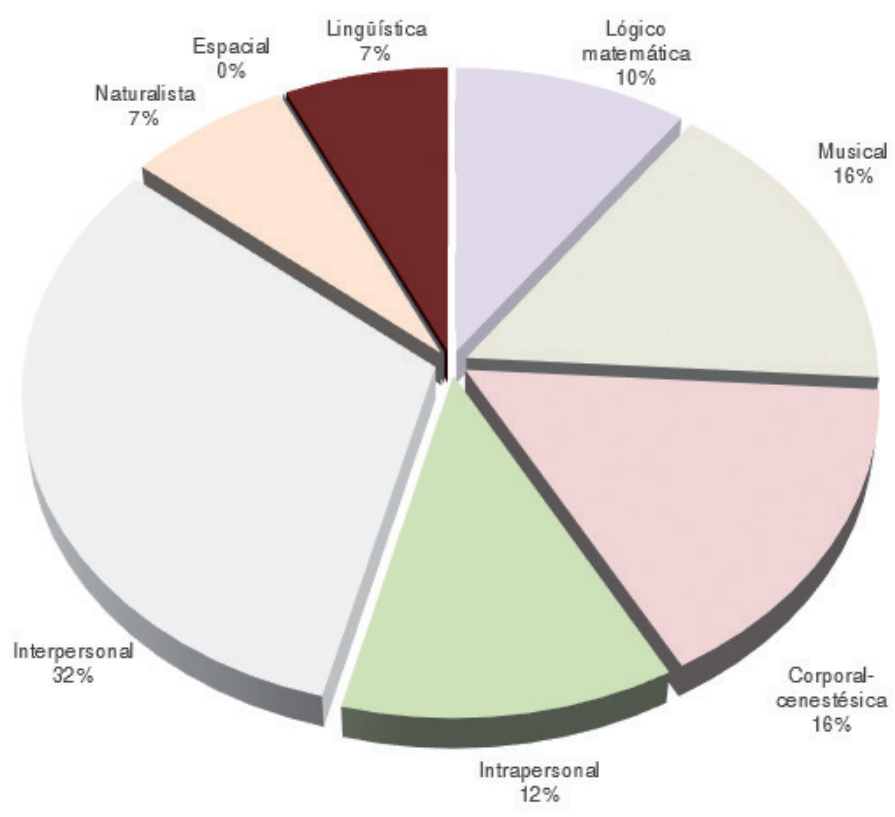

Figura 1. Resultados de la cuestión 4 en la que los alumnos/as definen su inteligencia más desarrollada (según las coordenadas de las IM de Gardner). 


\subsection{Desarrollo de la propuesta}

Como se ha mencionado, la propuesta proponía que cada participante desarrollara el proyecto en aquella inteligencia gardneriana en la que se encontrara más cómodo; esto es muy importante, porque, tal y como hemos visto en los primeros resultados del análisis contextual, ante una materia que se llame EVP los preconceptos de autoestima conducen con frecuencia al rechazo de la misma, por cuanto consideran no disponer de "habilidades suficientes".

Posteriormente, y a lo largo de tres semanas, los alumnos/as recibieron formación de técnicas artísticas diversas (entre las que se potenciaron las técnicas de creación volumétrica con materiales como el papel maché, la madera, la arcilla o el alambre así como las nociones sobre el color con técnicas como el gouache o el pastel, también se les enseñaron aquellas relacionadas con la imagen digital, la creación y modificación). Nos apresuramos a decir aquí que todo el sistema de técnicas se articulaba en una progresión de dificultad, desde técnicas artísticas más tradicionales a aquellas basadas en el empleo de los instrumentos digitales (retoque imagen fija, imagen en movimiento) que tenían como finalidad coadyuvar a crear un relato propio, guiándolos para que "aprendieran a sospechar" o a tener una actitud "sospechante" sobre las temáticas que querían trabajar en el proyecto final. Esto consiste en detenerse ante las imágenes y preguntarnos qué quieren decirnos, estableciendo un sistema de análisis sobre ellas y leerlas (Acaso, 2006). Todo ello debía integrarse en un proyecto final, para el cual se podía elegir cualquier tema utilizando cualquier técnica de las aprendidas, incluidas las digitales, con la intención, tal y como explica Wenham (2011), de ayudarnos a aprender cosas sobre nosotros mismos y sobre aquel modo que empleamos para relacionarnos con otras personas y con el mundo.

La idea era proporcionar al alumnado diversas oportunidades para que explorasen creativamente sus propios intereses y capacidades, siempre atendiendo al proceso de aprender habilidades y conceptos valiosos en el campo de la didáctica de la Expresión Plástica. Todo ello mediante diversos recursos multimodales, con la idea de desarrollar algunas de las IM.

El sistema de enseñanza aprendizaje diseñado partiendo de la teoría de proyectos hallaba su epílogo final en el hecho que el proyecto acababa siendo el elemento central y más importante de la evaluación 
establecida, ya que la relación entre método y evaluación, como hemos visto, es uno de los ejes estructurantes del planteamiento de Gardner.

De esta forma se pretendía que mediante caminos muy diversos cada alumno/a desarrollara sus potencialidades, no solo creativas, llegando a unos mismos conocimientos temáticos.

\subsubsection{EL PROYECTO FINAL}

El proyecto final se basaba en la idea que cada equipo de trabajo -formado por un máximo de cuatro personas- desarrollara materiales didácticos para la disciplina. Estos materiales podían referirse a la enseñanza reglada y también a la educación no formal, bien fuera relacionada con museos o otros equipamientos patrimoniales.

El planteamiento del proyecto final se quiso transmitir de forma interdisciplinar, para que aquellos integrantes del grupo decidieran aquella materia con la que se sentían más cómodos, así como aquella técnica que les permitía potenciar de mejor forma la propuesta con la inteligencia que tenían más desarrollada o que les interesaba investigar y desarrollar. Esta característica de la propuesta, pues, muestra cómo la intervención, pese a estar articulada desde una asignatura que pertenece al departamento de Didáctica de la Expresión Visual y Plástica, tiene la voluntad de ser un trabajo completamente interdisciplinar (Jové, Llonch, Bonastra, 2015).

Tabla 2. Tabla con la relación entre las propuestas y temas escogidos por el alumnado y las materias impartidas en primaria.

\begin{tabular}{ll}
\hline ÁREAS & TEMÀTICAS \\
\hline CCSS/ARTE & $>$ Tàpies para todos \\
& $>$ Guiñoles y arte \\
& $>$ Gaudí Experience \\
& $>$ Investiguemos en el Museo Víctor Balaguer \\
& $>$ Un robo en el Museo Nacional de Arte de Cata- \\
& lunya \\
& $>$ Barcelona Gótica y Modernista \\
& $>$ Mitología y arte \\
& $>$ Conozcamos el patrimonio del mundo \\
& $>$ El twistter de Barcelona \\
& $>$ Arte reciclado \\
& $>$ El arte revive \\
& $>$ Transformemos Mundet \\
&
\end{tabular}




\begin{tabular}{ll}
\hline ÁREAS & TEMÀTICAS \\
\hline HISTORIA & $>$ Las historias de Egipto \\
& $>$ Los huesos nos hablan \\
& $>$ El pasado de nuestro Campus \\
& $>$ La guerra de nuestros abuelos y bisabuelos \\
& $>$ Ciudad Inglesa \\
& $>$ Personajes ingleses \\
& $>$ Sherlock Holmes \\
& $>$ De ingleses y escoceses \\
\hline INGLÉS & $>$ Música y Arte \\
& $>$ ExpresiónArte (danza) \\
& $>$ InstumentArte \\
\hline MÚSICA & $>$ Las matemáticas pueden ser divertidas \\
& $>$ iLa soledad de los números primos? \\
\hline MATEMÁTICAS & $>$ La estética y el bosque \\
& $>$ Colores y formas en el mar \\
& $>$ Un diálogo entre paisaje y arte \\
\hline CIENCIAS NATURALES &
\end{tabular}

Así, tras la propuesta, los temas escogidos propuestos por el alumnado estuvieron basados en disciplinas distintas (Tabla 2), aunque una mayoría, dado que la propuesta se desarrollaba en la asignatura de EVP, estuvieron basadas en temáticas vinculadas con el arte. Así, se vieron propuestas sobre artistas; etapas históricas; percepción del medio urbano; museos; percepción del espacio geográfico; mitología; música y educación medioambiental. Es importante observar la diversidad en la elección de los temas, que, evidentemente, responde a intereses personales y, probablemente, a habilidades. Respecto a los formatos en que se presentaron los distintos proyectos, naturalmente respondían a las habilidades desarrolladas a lo largo del semestre y a las características propias de cada alumno/a.El proceso creativo se desarrolló principalmente en el aula, partiendo de la visualización y análisis de modelos propios de la museografía didáctica, siguiendo el siguiente esquema de las distintas fases: 


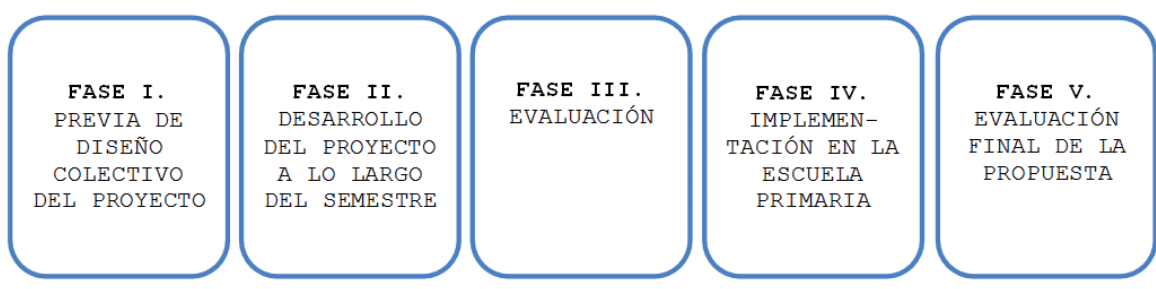

Figura 2. Las cinco fases de la que consta el planteamiento del trabajo por proyectos.

Como se ve (Fig. 2), la primera de las fases -previa de diseño colectivo del proyecto- es aquella que se basó en el conocimiento de modelos ya implementados, e incluyó la visita al gabinete didáctico del Museo Nacional de Arte de Catalunya (MNAC) para que el alumnado pudiera ver en acción materiales diseñados por profesionales. La segunda de las etapas consistió en desarrollar a lo largo de las sesiones de un semestre universitario aquellas actividades en el aula que les hicieron más doctos/ as en las estrategias y en el manejo de los materiales y técnicas artísticas con el fin de que fueran capaces de desarrollar sus propios materiales didácticos para el proyecto didáctico. La fase de evaluación consistió en la evaluación por parte de la docente, así como de la técnica en educación del MNAC; todo ello, complementado con la puesta en marcha del material didáctico tanto en el aula como en el museo. Finalmente solo Ilegaron a la última fase aquellos grupos que entre el propio alumnado se habían elegido como las propuestas más adecuadas para quedar establecidas como propuesta didáctica ante un grupo clase real de primaria de la Escuela Miquel Bleach (Barrio de Sants en Barcelona).

\subsubsection{La eVAluación}

Como hemos visto en el marco teórico, el planteamiento, la evaluación en el caso de las IM pasa por que alumno/a sea evaluado según su propio perfil, una propuesta muy sugestiva siempre que se mueva en el marco estricto del sentido común, dado que un modelo de este tipo no es de fácil aplicación sin recursos económicos importantes.En este sentido, es interesante detenerse en la fase cuarta y quinta por la relevancia de las mismas. Los materiales didácticos construidos fueron presentados en el aula y testados brevemente por el resto de discentes. Es en este momento cuando se perciben factores muy difíciles de evaluar-aunque se utilizaron plantillas de observación- como son el entusiasmo del equipo 
y el que se despierta en el resto de grupo-clase, las capacidades comunicativas, la pulcritud en la presentación, la aplicabilidad de los materiales y, en definitiva, la creatividad.

Después de llevar a cabo todo ello y a través de la experimentación presentada, se propone el diseño de modelo recogido en la Fig. 3 en el que se basaría toda la propuesta y donde vemos como aparecen dos grandes zonas diferenciadas (teoría/práctica); en la práctica se representa al sujeto (estudiante) qua ante un elemento patrimonial cualquiera y a través de la asimilación de técnicas con el objetivo de la creación de material didáctico llega a la interpretación del elemento inicial. La parte más teórica es donde podemos ver como a través de las inteligencias múltiples se potencian las propias habilidades y competencias a la vez que se refuerzan las potencialidades intelectuales.

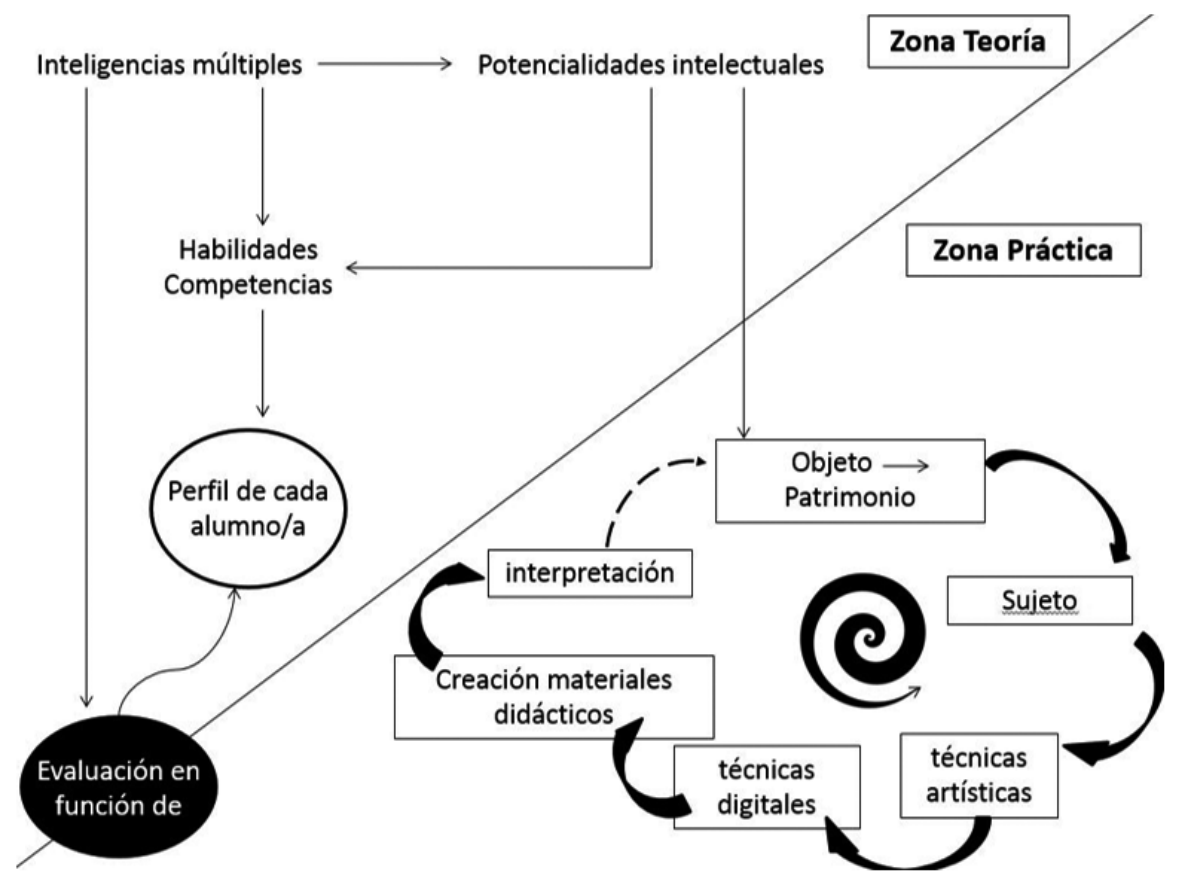

Figura 3. Planteamiento del modelo. 


\section{Resultados y discusión}

Los principales resultados de la investigación son los siguientes:

a) La estrategia empleada generó una extraordinaria diversificación temática y metodológica entre los participantes; aunque el modelo de partida fue el visualizado y experimentado en el MNAC, la producción egresada de los tres grupos difirió notablemente del modelo como es evidente analizando la tabla número 2 .

b) El segundo resultado se visualizó en el campo de la creatividad, ya que al explorar cada participante sus propias habilidades intelectuales, las ideas originales e innovadoras fueron muy superiores a lo que es habitual en este tipo de grupos. En una futura fase se creará un test específico para medir este resultado ya que se desestimaron los test más utilizados en el área como el Test de Pensamiento Creativo (Torrance, 1962), el Test TAEC (De la Torre, 1996) o el Test CREA (Corbalán, Martínez, Donolo 2003) porque tendían a analizar aquellos aspectos relacionados con la creatividad espacial, gráfico o verbal, parámetros no destacables en las propuestas.

c) El tercer resultado fue el desarrollo de propuestas de carácter transversal con otras disciplinas, desde la botánica a las matemáticas o a la historia.

Estos resultados parecen claros indicadores de que, sin discutir el valor epistemológico de la teoría de las IM, su aplicación en nuestro campo es fértil por cuanto sugiere elementos innovadores, transversales y extraordinariamente diversificados.

\section{Conclusiones}

Antes de iniciar las conclusiones queremos recordar que tenemos la voluntad de que la presente experiencia se inscriba dentro de las líneas que trabajan en didáctica e interpretación del Patrimonio promovidas desde el área de Didáctica de la Expresión Plástica; entre ellas podemos ver como existe un replanteamiento acerca de conceptos que se pueden trabajar desde el área, como es la didáctica del patrimonio. Creemos pues que, es un buen momento para definir nuevos modelos para la didáctica 
del patrimonio desde la educación artística, en que se tengan en cuenta la dimensión artística y tecnológica.

Nuestros objetivos iniciales consistían, como se recordará, en detectar las motivaciones del alumnado que cursa la asignatura de Didáctica de la EVP en la Facultad de Educación como asignatura obligatoria del Grado en Primaria y, por otra parte, en consolidar una estrategia metodológica a través de una intervención didáctica para el desarrollo de la capacidad artística entre las IM y la enseñanza de las Artes Visuales a través de la didáctica del patrimonio.

Respecto a al primer objetivo los resultados nos han mostrado que las inquietudes de los alumnos/as se centran en aprender cómo enseñar en sus futuras aulas y cómo combatir el miedo al desconocimiento a través de una buena metodología (cómo enseñar, qué herramientas y materiales utilizar, para qué y por qué). Con el presente proyecto se ha pretendido dotar al alumnado de una metodología que unifica las herramientas de la didáctica del patrimonio con la didáctica de la expresión visual y plástica mostrando como la interrelación es fructífera. Hay que tener en cuenta que también el alumnado manifiesta el interés por el desarrollo y elaboración de proyectos, así como un gran interés en saber cómo plantear y crear materiales didácticos con una especial voluntad de transversalidad entre materias. Con esta propuesta se buscaba consolidar una estrategia metodológica a través de una intervención didáctica para el desarrollo de la capacidad artística entre las IM y la enseñanza de las Artes Visuales.

Obviamente, aun cuando los objetivos se cumplieron y hoy conocemos algo más sobre las motivaciones de nuestros estudiantes, ésta experiencia plantea de nuevo la necesidad de interacción entre el área de Didáctica de la Expresión Plástica y el área de Didáctica de las Ciencias Sociales así como la efectividad de la aplicación de la teoría de las IM en otros campos como los referidos modelizando experiencias como la presente.

\section{Referencias bibliográficas}

Allport, G. (1959). Personalidad: una interpretación psicológica. Buenos Aires: Paidós. Acaso, M. (2006). Esto no son las Torres Gemelas. Cómo aprender a leer la televisión y otras imágenes. Madrid: Catarata.

Acaso, M. (2009). La educación artística no son manualidades. Nuevas prácticas en la enseñanza de las artes y la cultura visual. Madrid: Catarata. 
Una experiencia de formación del profesorado basada en las inteligencias múltiples y

la Educación Artística

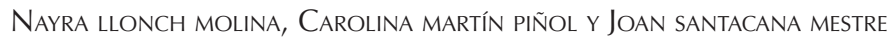

Acaso, M. (2013). rEDUvolution: hacer la revolución en la educación. Barcelona: Paidós.

Báez, C. (2010). La educación plástica hoy: pensamiento del profesorado y propuestas de intervención en la escuela primaria. Actas // CIDd Congrés Internacional de Didàctiques, 3-6 de febrero, Girona.

Beetlestone, F. (2004). Niños creativos, enseñanza imaginativa. Madrid: La Muralla.

Corbalán, F.J., Martínez, F. y Donolo, D. (2003). CREA. Inteligencia creativa. Una medida cognitiva de la creatividad. Madrid: Trea ediciones.

De La Torre, S. (1996). TAEC: Test de Abreación para la Evaluación de la Creatividad. Madrid: Escuela Española.

Efland, A. D. (2002). Art and cognition: Integrating the visual arts $n$ the curriculum. New York: Teachers College Press \&Reston, A: National Art Education Association.

Eisner, E. (2004). El arte y la creación de la mente. El papel de las artes visuales en la transformación de la consciencia. Barcelona: Paidós Ibérica.

Fodor, A. (1983). The Modularity of Mind: An Essay in Faculty Psychology. Cambridge, MA: MIT Press.

Fontal, O., Marín, S. y García, S. (2015). Educación de las Artes Visuales y Plásticas en Educación Primaria. Madrid: Colección Didáctica y desarrollo.

Fontal, O. (2008). Hacia una educación artística "patrimonial". En I. Aguirre, O. Fontal, B. Darrás, B y R. Rickenmann (coords.), El acceso al patrimonio cultural. Retos y Debates. Pamplona: Universidad Pública de Navarra y Cátedra Jorge Otiza.

Fontal, O. (2008). La importancia de la dimensión humana en la Didáctica del Patrimonio. En S.M. Mateos (Coord.), La comunicación global del patrimonio cultural (pp.79-109). Gijón: Trea.

Gardner, H. (1994). Estructuras de la mente. La teoría de las inteligencias múltiples. México: S.L. Fondo de Cultural Económica de España.

Gardner, H. (2003). La inteligencia reformulada: las inteligencias múltiples en el siglo XXI. Barcelona: Paidós Ibérica.

Gardner, H. (2010). Mentes creativas. Una anatomía de la creatividad. Barcelona: Paidós.

Gómez Redondo, C. (2013). Procesos de patrimonializaciónen el arte contemporáneo: diseño de un artefacto educativo para la identización (Tesis doctoral inédita). Valladolid: Universidad de Valladolid.

Hernández, F. (2008). La investigación basada en las artes. Propuesta para repensar la investigación en educación. Educatio siglo XXI (26), 85-118.

Ibáñez, C. (2010). La teoría de las inteligencias múltiples: algunos énfasis críticos. Revista Mexicana de Investigación en Psicología, 2(2), 136-140.

Jové, G., Llonch, N. y Bonastra, Q. (2015). (Inter)(Trans)disciplinariedad en formación de maestros. Aula de Innovación Educativa, 239, 16-22.

Larivée, S. (2010). Las inteligencias múltiples de Gardner. ¿Descubrimiento del siglo o simple rectitud política? Revista Mexicana de Investigación en Psicología, 2(2), 115-126.

Larenas, B. (2005). Didáctica de las Artes Visuales sustentada en la propuesta de las inteligencias múltiples de Howard Gardner: experiencia aplicada en un primer año y medio de la comuna de concepción. Revista Ingenieria Industrial, 4 (1), 73-87.

Levy, P. (2008). Method meets art: arts-based research practice method meets art, New York: Guilford Press. 
Una experiencia de formación del profesorado basada en las inteligencias múltiples y

la Educación Artística

Nayra llonch molina, Carolina martín piñol y JoAn SantaCana mestre

Luca, S.L. de (2000). El docente y las inteligencias múltiples. Revista Iberoamericana de Educación, 342, 1-11. Recuperado de http://www.rieoei.org/deloslectores/616Luca. PDF

Lopez, M. (2015). Para qué el arte. Reflexiones en torno al arte y su educación en crisis. Madrid: Fundamentos.

Morales, J.J. (2001). La evaluación en el área de educación visual y plástica en la educación secundaria obligatoria (Tesis Doctoral). Recuperado de http://www.tesisenred. net/bitstream/handle/10803/5036/jjma01 de16.pdf.pdf?sequence=1

Muset, M. (2001). Ovide Decroly: la pedagogía de los centros de interés y de los métodos globales. En J. Trilla (coord.), El legado pedagógico del siglo XX para la escuela del siglo XXI, p. 95-122. Barcelona: Graó.

Piaget, J. (1961). La Formación del Símbolo en el Niño: imitación, juego y sueño, imagen y representación. México: Fondo de Cultural Económica.

Platón (2003). Diálogos. Obra completa en 8 volúmenes. Vol. II y IV. Madrid: Gredos.

Santacana, J. yY Coma, L. (2014). El m-learning y la educación patrimonial. Gijón: Trea.

Santacana, J. y López, V. (2015). Educación, tecnología digital y patrimonio cultural. Para una educación inclusiva. Gijón: Trea.

Suazo, S. N. (2010). Inteligencias múltiples. Manual práctico para el nivel elemental. San Juan: Universidad de Puerto Rico.

Suárez, J., Maiz, F. y Meza, M. (2010). Inteligencias múltiples: una innovación pedagógica para potenciar el proceso de enseñanza aprendizaje. Investigación y Posgrado, 25(1), 81-94.

Torrance, E. P. (1974). The Torrance test of creative thinking: Norms-technical manual. Illinois: Scholastic Testing Service.

Wenham, M. (2011). Entender el arte. Una guía para el profesorado. Barcelona: Graó. 